\title{
Ultrasound Accuracy in Confirming Cuffed Endotracheal Tube Position for Pediatric Intensive Care Unit Patients
}

\author{
Nabeel Almashraki ${ }^{1}$, Sawsan Alyousef ${ }^{1,2^{*}}$, Mustafa Safi ${ }^{1}$, Nabil Sadiq ${ }^{1}$, Shamaila Amjad ${ }^{1}$, Ahmad A. Al Boukai ${ }^{3}$ and Jihad \\ Zahraa $^{1}$ \\ ${ }^{1}$ Pediatric Intensive Care Department, Specialized Children Hospital, King Fahad Medical City, Riyadh, Saudi Arabia \\ ${ }^{2}$ Center for Research, Education \& Simulation Enhanced Training (CRESENT), King Fahad Medical City, Riyadh, Saudi Arabia \\ ${ }^{3}$ Radiology Department, King Khalid University Hospital, Riyadh, Saudi Arabia
}

Abstract
Objective: To compare the accuracy of ultrasound (US) in assessing cuffed endotracheal tube (ETT)
position using the suprasternal visualization approach versus chest radiography for intubated Pediatric
Intensive Care Unit (PICU) patients.

Methods: A prospective study was conducted between October 2014 and March 2016 on sixty-six intubated patients less than 15 years of age who met predetermined inclusion criteria. Suprasternal tracheal ultrasound was done to visualize the ETT cuff filled with saline before obtaining the routine chest radiography, the gold standard used to determine ETT position and depth. The results of two methods Publication History: Received: January 06, 2018

Accepted: February 21, 2018

Published: February 23, 2018

\section{Keywords:}

Critically ill children, Chest X-ray, saline-filled ETT cuff were compared.

Results: US examination to detect the proper position of ETT as compared to chest radiography revealed a sensitivity of $91.67 \%$ (95\% CI, $80.02 \%$ to $97.68 \%$ ), specificity of $83.33 \%$ (95\% CI, $58.58 \%$ to $96.42 \%$ ), positive predictive value of $93.62 \%$ ( $95 \% \mathrm{CI}, 82.46 \%$ to $98.66 \%$ ), negative predictive value of $78.95 \%$ (95\% CI, $54.43 \%$ to $93.95 \%$ ), positive likelihood ratio of 5.50 (95\% CI 1.95 to 15.51 ), and Negative Likelihood Ratio of 0.10 (95\% CI 0.04 to 0.26 ).

Conclusion: Ultrasonography was found to be a more feasible, safer, and relatively quicker alternative method to determine the proper position of ETT in the trachea of PICU patients when using salinefilled ETT cuff with high sensitivity and specificity. Larger controlled studies are needed to support generalizability.

\section{Introduction}

Endotracheal intubation is a commonly performed invasive procedure in the Pediatric Intensive Care Unit (PICU) worldwide. Accurate assessment of the endotracheal tube (ETT) position or depth is critical to prevent possible wrong-placement-associated complications and morbidities [1,2].

There are multiple methods used to confirm proper placement of ETT, being endotracheal versus wrongly placed in the esophagus such as direct visualization, clinical assessment of air entry, and $\mathrm{CO} 2$ detector. Other methods are used to confirm proper position (i.e. depth) of the ETT in the trachea like lung auscultation, chest radiograph, and fiberoptic bronchoscopy [3,4]. The current gold standard test to confirm proper ETT position in critically ill patients is chest radiography; however, this is often done later after ventilation has commenced. Performing chest radiography also requires patient manipulation and X-ray film positioning that may be associated with a risk of ETT displacement and even dislodgement [5].

Another limitation is encountered during Cardiopulmonary Resuscitation (CPR), which makes chest radiography impractical, as it requires the interruption of chest compressions [6]. Adding to the above, there is also the hazard of repeated radiation exposure in severely ill patients [7].

Ultrasound (US) applications in medicine have been expanding remarkably. In addition to its well-established role in diagnostic and therapeutic interventions, ultrasound has been successfully used in several airway-related applications. It has been used to confirm ETT placement and depth/position, assess vocal cord movement, and diagnose pneumothorax and others [8-12]. Using
US to directly visualize the ETT in the trachea is technically difficult due to acoustic impedance of the air; therefore, several studies used indirect ultrasound signs of correct ETT depth like bilateral lung sliding and diaphragm movement [13-15]. This was found to be not very reliable when compared to chest radiography [16].

To date, few studies have evaluated the value of US technique in visualizing a saline-filled cuff in intubated patients. They were conducted on adults, cadaveric model and in children undergoing general anesthesia for elective surgery $[17,18]$.

Our study main objective is to compare the accuracy of assessing ETT depth by suprasternal US visualization of saline filled cuff versus Chest radiography for critically ill, intubated PICU patients. To our knowledge, our study is the first to use such techniques in these settings.

\section{Methods}

This was a prospective study conducted from October 2014 to March 2016 in the PICU at King Fahad Medical City, a tertiary care center located in Riyadh, Saudi Arabia. We obtained an approval from our Institutional Review Board before starting patient recruitment.

"Corresponding Author: Prof. Sawsan Alyousef, Center for Research, Education \& Simulation Enhanced Training (CRESENT), King Fahad Medical City, Riyadh Saudi Arabia; E-mail: salyousef@kfmc.med.sa

Citation: Alyousef S, Almashraki N, Safi M, Sadiq N, Amjad S, et al. (2018) Ultrasound Accuracy in Confirming Cuffed Endotracheal Tube Position for Pediatric Intensive Care Unit Patients. Int J Pediatr Neonat Care 4: 135. doi: https://doi.org/10.15344/2455-2364/2018/135

Copyright: (c) 2018 Alyousef, et al. This is an open-access article distributed under the terms of the Creative Commons Attribution License, which permits unrestricted use, distribution, and reproduction in any medium, provided the original author and source are credited. 
Citation: Alyousef S, Almashraki N, Safi M, Sadiq N, Amjad S, et al. (2018) Ultrasound Accuracy in Confirming Cuffed Endotracheal Tube Position for Pediatric Intensive Care Unit Patients. Int J Pediatr Neonat Care 4: 135. doi: https://doi.org/10.15344/2455-2364/2018/135

Page 2 of 5

The study population consisted of a convenience sample of PICU patients less than 15 years of age who were recently or previously intubated using a cuffed ETT.

We excluded from the enrolment high risk patients who could be affected by losing lung recruitment during cuff deflation such as patients who are on high frequency ventilation, requiring positive end expiratory pressure $(\mathrm{PEEP}) \geq 10 \mathrm{~cm} \mathrm{H} 2 \mathrm{O}$, with severe pulmonary hypertension, active pulmonary hemorrhage, and patients with high Intracranial pressure (ICP). We also excluded patients with neck masses, scars, or with difficult airway due to difficulties in performing the US studies on them. An informed consent was obtained from patient's parents or guardians before enrollment in the study.

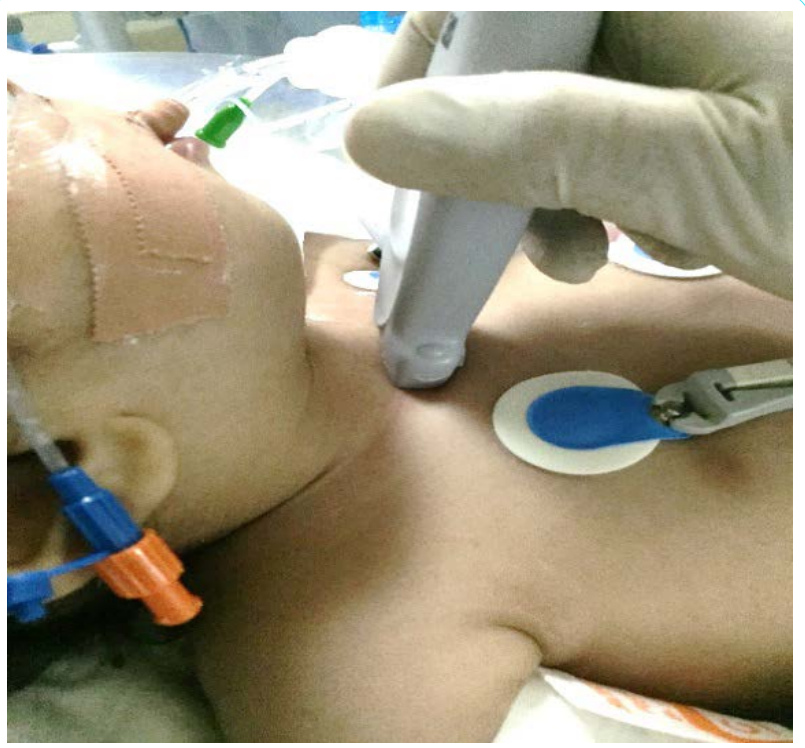

Figure 1: Probe placement while patient head in neutral Position.

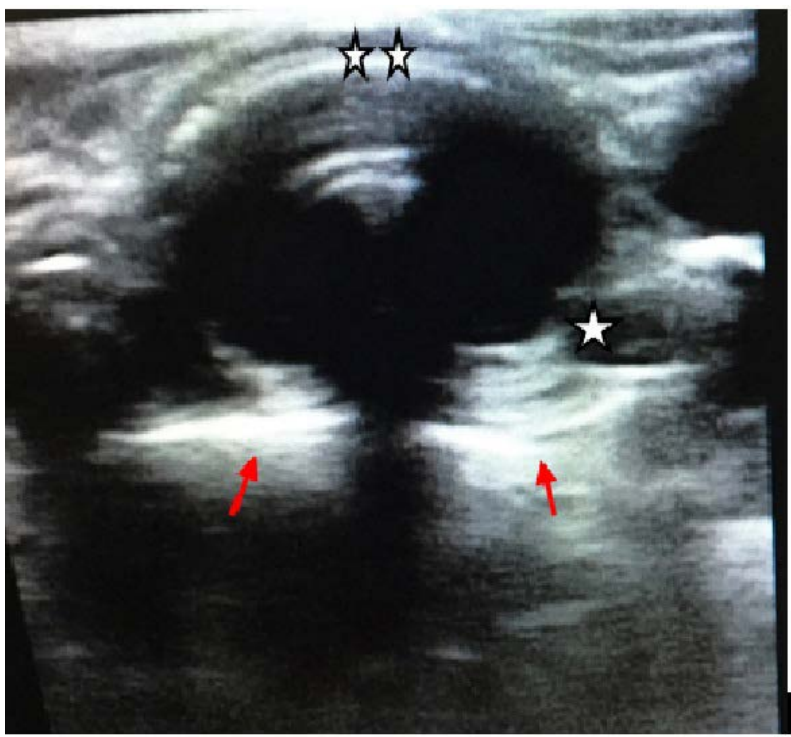

Figure2: Ultrasound image demonstrating shadows representing saline-filled cuff of endotracheal tube.

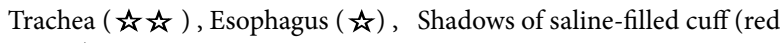
arrows).
Endotracheal tube brand used was Portex (Smiths Medical) made from Polyvinylchloride (PVC), with low pressure-high volume cuff type, and inner diameter range from 3 to $6 \mathrm{~mm}$ based on our guidelines using international references [19].

Ultrasound scan of the airway using a linear array probe positioned just above the suprasternal notch was done to a convenient sample of freshly intubated or already intubated patients before the assessment of a chest radiography that is usually requested as part of routine PICU care. The US study should have no negative impact or cause any delay on the patient's scheduled care.

This visualization of airway by US was conducted by a well-trained PICU physician who has received an extensive training (structured courses for emergency and critical care US) and has been using US in the daily PICU practice for more than two years.

The position of ETT as determined by the US technique was compared with the position from the chest radiography taken shortly after the US study. We used the following definitions of Ultrasound ETT tip position for all of our study's population: Proper ETT position was defined as follows: The cuff shadows appear with the probe level just above suprasternal notch and below thyroid cartilage. Improper ETT position was observed when cuff shadows appear with the probe level at or above thyroid cartilage (high) or when cuff shadows did not appear on US screen (deep) [18].

For patient chest radiography, the Pediatric Intensivist on duty looked at the film and defined the proper vs improper ETT position as follows: Proper ETT level was when the ETT tip is seen below first thoracic vertebra (T1) and at or above the third thoracic vertebra (T3). Improper ETT level was when the ETT tip was seen at or above T1 (high) or below the body of the third thoracic vertebrae (T3) (deep) provided that carina was identified at $\mathrm{T} 4$ or lower [20-22].

An expert pediatric radiologist who was blinded to the US results and the pediatric intensivist readings have assessed the chest radiographies independently.

\section{Ultrasound Machine and Technique}

We used high frequency $(\mathrm{MHz} 10-13)$ linear array probe of the M-Turbo ultrasound machine (SonoSite, Inc, Bothell, WA) for all studies.

\section{Technique}

With the patient lying supine and in the neutral position, the examiner checked the integrity of the ETT cuff then deflated it using a $5 \mathrm{~mL}$ syringe, measuring the volume of air removed. He then reinflated the ETT cuff with the same volume using normal saline while utilizing a manometer to ensure the cuff pressure stayed within the acceptable values of 20-30 mmHg. After completing the above step, the examiner stood on the patient's right side and palpated the sternal bone. He moved the fingers towards the patient's head and located the suprasternal notch. He then placed a high-frequency linear probe in transverse position and perpendicular to the anterior neck just above the suprasternal notch as shown at (Figure 1). If the cuff was not seen, he would sweep the probe superiorly along the trachea to the level just above the thyroid cartilage to search for the saline-inflated cuff. 
At the end of the scanning, the physician would record on a data collection sheet the patient's demographics, scan duration, any change in patient status, unexpected events during procedure and his US image interpretation. After obtaining the chest radiography, the examiner would record the ETT depth on the film as interpreted by the managing PICU team. All participants have received standard PICU care, and no patient care decisions have been made based upon the findings of the ultrasound studies.

\section{Statistical Analysis}

The estimation of sample was determined by PASS 11 software (Hintze, J. 2011). A sample size of 66 subjects achieved 90\% power at a 5\% significance level using one-sided equivalence test of correlated proportions and $88 \%$ expected outcome of suprasternal ultrasound accuracy of assessing ETT depth was considered with maximum allowable difference between these proportions that still results in equivalence (the range of equivalence) is 0.14 .

All Categorical variables such as gender presented as numbers and percentages. Continuous variables such as age, weight, height and Body Mass Index (BMI) were expressed as median and interquartile range. We used Chi-square or Fisher's exact test to determine the significant relationship among categorical variables. Diagnostic test analysis was applied to determine the sensitivity, specificity, PPV and NPV of the ultrasound accuracy test compared with chest radiograph test. P-value of less than 0.05 was considered as statistically significant. All data were entered and analyzed using statistical package SPSS version 22 .

\section{Results}

Between October 2014 and March 2016, 66 eligible patients consented and were enrolled in our study. The mean age was 43.68 months while median was 16 months (with IQR of 62) (Table 1) and 37 (56.06\%) were males and 29 (43.93\%) were females.

\begin{tabular}{|l|l|l|l|l|}
\hline Characteristics & Minimum & Maximum & Median & IQR (Q3 - Q1) \\
\hline Age $($ Months $)$ & 0.13 & 180.00 & 16.0000 & 62 \\
\hline Weight $(\mathrm{kg})$ & 2.30 & 48.00 & 8.0000 & 9.50 \\
\hline Height $(\mathrm{cm})$ & 43.00 & 155.00 & 76.0000 & 32 \\
\hline BMI & 9.58 & 22.68 & 13.4930 & 3.09 \\
\hline
\end{tabular}

Table 1: The demographics and clinical characteristics of study subjects $\mathrm{BMI}$, body mass index; IQR, interquartile range.

Endotracheal tube position determined by Ultrasound was proper in $47(71.21 \%)$ cases, and improper in $19(28.78 \%)$ cases, and non showed oesophageal intubation, while endotracheal tube position as determined by chest radiography was proper in 48 (72.72\%), and improper in $18(27.27 \%)$ cases as presented in (Table 2$)$.

\begin{tabular}{|l|l|l|l|l|}
\hline Technique & & \multicolumn{2}{|l|}{ Chest Radiograph } & \\
\hline & & Proper & Improper & Total \\
\hline \multirow{3}{*}{ Ultrasound } & Proper & 44 & 3 & $47(71.21 \%)$ \\
\cline { 2 - 5 } & Improper & 4 & 15 & $19(28.78 \%)$ \\
\cline { 2 - 5 } & Total & $48(72.72 \%)$ & $18(27.27 \%)$ & 66 \\
\hline
\end{tabular}

Table 2: Comparing endotracheal tube (ETT) position by ultrasound and chest radiograph $(\mathrm{n}=66)$

Kappa $=0.737-$ p-value $<0.001$.
US examination to detect proper position of ETT as compared to chest radiography revealed a sensitivity of $91.67 \%$ (95\% CI, $80.02 \%$ to $97.68 \%$ ), specificity of $83.33 \%$ (95\% CI, $58.58 \%$ to $96.42 \%$ ), positive predictive value of $93.62 \%$ (95\% CI, $82.46 \%$ to $98.66 \%$ ), negative predictive value of $78.95 \%$ (95\% CI, $54.43 \%$ to $93.95 \%)$, positive likelihood ratio of 5.50 ( $95 \%$ CI 1.95 to 15.51), and negative likelihood ratio of 0.10 (95\% CI 0.04 to 0.26 ), as presented in (Table 3)

\begin{tabular}{|l|l|l|}
\hline Statistic & Value & $95 \%$ CI \\
\hline Sensitivity & $91.67 \%$ & $80.02 \%$ to $97.68 \%$ \\
\hline Specificity & $83.33 \%$ & $58.58 \%$ to $96.42 \%$ \\
\hline Positive Predictive Value & $93.62 \%$ & $82.46 \%$ to $98.66 \%$ \\
\hline Negative Predictive Value & $78.95 \%$ & $54.43 \%$ to $93.95 \%$ \\
\hline Positive Likelihood Ratio & 5.50 & 1.95 to 15.51 \\
\hline Negative Likelihood Ratio & 0.10 & 0.04 to 0.26 \\
\hline
\end{tabular}

Table 3: Accuracy of detecting proper position of endotracheal tube (ETT) by ultrasonography in comparison with chest radiograph. CI: confidence interval.

The overall accuracy of ultrasonography in confirmation of ETT position based on the area under the receiver operating characteristic (ROC) curve was $89.40 \%$ (figure 3 ). None of the sixty-six patients developed any complications during the procedure.

There was an agreement in $96 \%$ of the cases between the radiologist and intensivist ETT position assessment. The median time for the US procedure was 5 minutes, with IQR of 1 minute, while the median time for the Chest radiography procedure was 30 minutes with IQR of 10 .

\section{Discussion}

Malposition of ETT is common and considered as an important complication of endotracheal intubation in pediatrics. Hence, wrong positioning, especially main stem bronchus intubation, needs to be quickly detected and corrected. Determining the accurate depth of the ETT in critical care areas is still almost fully dependent on chest radiography with all its' potential risks and delays. Other moreaccurate methods have been used, like fiberoptic bronchoscopy which is, however, an invasive procedure, not readily available and requires specialized training. Bedside Ultrasonography is frequently used in critical care areas for different types of emergencies and elective procedures, as it is proved to be rapid, safe, portable, reproducible, relatively inexpensive and not difficult to master. Nowadays, this technique is being utilized more in airway related procedures and ETT detections in all age groups [23].

There have been a few small studies that described the use of US technique to detect proper placement and depth of ETT in adult and pediatric ICU patients with reasonable sensitivity and specificity. These studies have either used the diaphragm movement and lung sliding, or direct visualization of the ETT tip as a surrogate marker of proper ETT depth and placement $[23,13]$.

ETT could be easily visualized in the majority of patients included in this study by ultrasonography using the saline-filled ETT cuff. It has shown superior sensitivity and specificity than the previously described methods. A somewhat similar study was conducted by Tessaro, et al. (trust study) on pediatric elective surgery patients and showed a high sensitivity (98.8\%) and specificity (96.4\%) of 


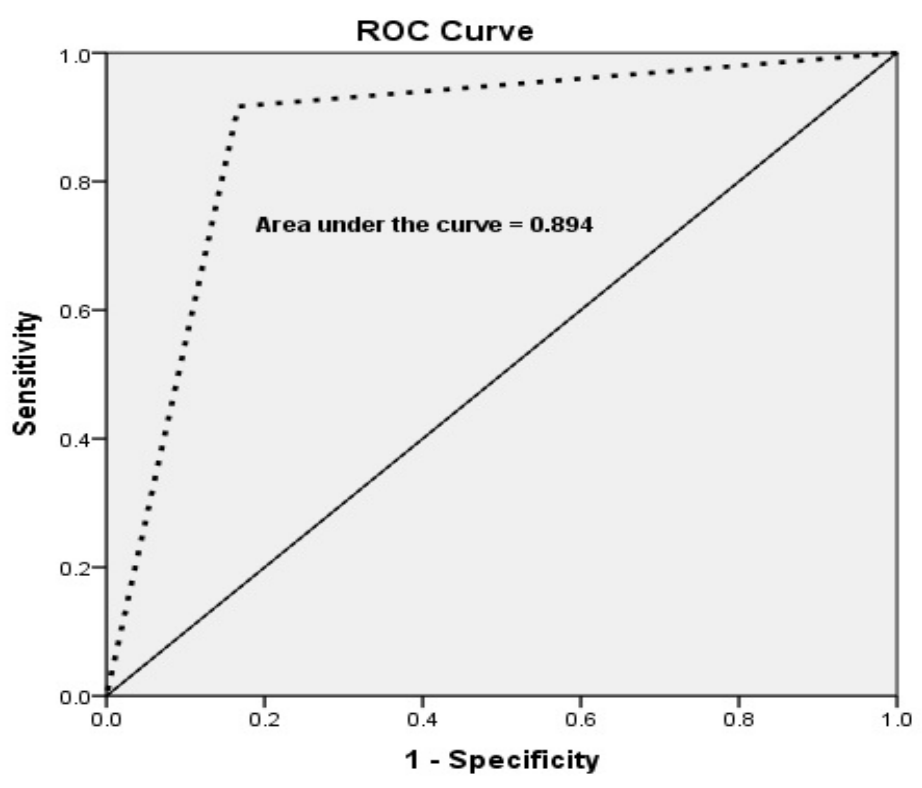

Diagonal segments are produced by ties.

Figure 3: Receiver operating characteristic curve (ROC) of ultrasound accuracy in detecting cuffed endotracheal tube position.

the US technique in detecting proper position of saline-filled cuffed ETT [18]. The team used bronchoscopy examination as reference for comparison as all their patients were clinically stable, intubated and in a well-controlled environment in operating room. Our study showed somewhat less sensitivity $(91.67 \%)$ and specificity (83.33\%) but it was conducted on real PICU critically ill patients and used chest radiograph, the standard methodology in ICUs, as the reference. The detection of ETT position using US is not a complex procedure and can be learned and performed by PICU physicians after receiving the proper training. A group of investigators from Texas were able to demonstrate using cadaveric model that a novice pediatric emergencyfellow who received around 50 minutes of training achieved up to $96 \%$ sensitivity [17]. Filling up the ETT cuff has probably contributed to the clarity and accuracy of detecting the tube position with less impact of air shadowing on image quality, it is a safe practice that is commonly used in certain pediatric laryngeal procedures $[24,25]$.

Although our study is the first to describe this technique in real PICU patients, it has few limitations like the small number of patients, the exclusion of many cases like very critically ill patients and difficult intubation cases, using only one ETT brand, and having only one experienced operator for all described cases. We do not believe the last limitation will have a major impact on generalizability of this procedure as the simplicity and ease of this technique is very encouraging and we are hoping larger sample size and multiple operators in a multicenter study will confirm this further.

\section{Conclusion}

Ultrasonography was found to be more feasible, safer, and relatively quicker alternative method to determining the proper position of ETT in the trachea of PICU patients when using saline-filled ETT cuff with relatively high sensitivity and specificity. Larger controlled studies are needed to support generalizability of this promising technique.

\section{Competing Interests}

The authors declare that they have no competing interests

\section{Acknowledgement}

To all PICU respiratory therapist and Nurses and the Secretary Ms Myra Verano, the statistician Mohammed Salman Bashir at KFMC.

\section{References}

1. Griesdale DE, Bosma TL, Kurth T, Isac G, Chittock DR, et al. (2008) Complications of endotracheal intubation in the critically ill. Intensive care medicine 34:1835-1842.

2. Jaber S, Jung B, Chanques G (2009) Endotracheal Intubation in the ICU Yearbook of Intensive Care and Emergency Medicine 313-321.

3. Hemmerling TM, Giacalone M (2016) Ultrasound and airway management. In: Ultrasound in Anesthesia, Critical Care and Pain Management with Online Resource 2nd ed. Cambridge University Press 7: 130-133.

4. Hagberg CA (2013) Monitoring the Airway and Pulmonary function. In: Benumof and Hagberg's Airway Management. 3rd edition Philadelphia, Elsevier Saunders 49: 1000-100.

5. Menon K, Dundon B, Twolan BL, AIShammari S (2015) Approach to unplanned extubations in a pediatric intensive care unit. Can J Crit Care Nurs 26: 25-9.

6. Brouwer TF, Walker RG, Chapman FW, Koster RW (2015) Association Between Chest Compression Interruptions and Clinical Outcomes of Ventricular Fibrillation Out-of-Hospital Cardiac Arrest. Circulation 132: 1030-1037.

7. Bartley K, Metayer C, Selvin S, Ducore J, Buffler P, et al. (2010) Diagnostic X-rays and risk of childhood leukaemia. International Journal of Epidemiology 39: 1628-1637.

8. Muslu B, Sert H, Kaya A, Demircioglu RI, Gözdemir M, et al. (2011) Use of sonography for rapid identification of esophageal and tracheal intubations in adult patients. J Ultrasound Med 30: 671-676.

9. Chou HC, Tseng WP, Wang CH, Ma MH, Wang HP, et al. (2011) Tracheal rapid ultrasound exam (T.R.U.E.) for confirming endotracheal tube placement during emergency intubation. Resuscitation 82: 1279-1284. 
Citation: Alyousef S, Almashraki N, Safi M, Sadiq N, Amjad S, et al. (2018) Ultrasound Accuracy in Confirming Cuffed Endotracheal Tube Position for Pediatric Intensive Care Unit Patients. Int J Pediatr Neonat Care 4: 135. doi: https://doi.org/10.15344/2455-2364/2018/135

Page 5 of 5

10. Sağlam C, Unlüer EE, Karagöz A (2013) Confirmation of endotracheal tube position during resuscitation by bedside ultrasonography. Am J Emerg Med 31: $248-250$

11. Galicinao J, Bush AJ, Godambe SA (2007) Use of bedside ultrasonography for endotracheal tube placement in pediatric patients:a feasibility study. Pediatrics 120: 1297-1303

12. Marciniak B, Fayoux P, Hébrard A, Krivosic-Horber R, Engelhardt T, et al (2009) Airway management in children: ultrasonography assessment of tracheal intubation in real time? Anesth Analg 108: 461-465.

13. Lin MJ, Gurley K, Hoffmann B (2016) Bedside Ultrasound for Tracheal Tube Verification in Pediatric Emergency Department and ICU Patients: A Systematic Review. Pediatr Crit Care Med 17: e469-e476.

14. Sim SS, Lien WC, Chou HC, Chong KM, Liu SH, et al. (2012) Ultrasonographic lung sliding sign in confirming proper endotracheal intubation during emergency intubation. Resuscitation 83: 307-312.

15. Hsieh KS, Lee CL, Lin CC, Huang TC, Weng KP, et al. (2004) Secondary confirmation of endotracheal tube position by ultrasound image. Critical Care Med 32: 374-377.

16. Kerrey BT, Geis GL, Quinn AM, Hornung RW, Ruddy RM, et al. (2009) A prospective comparison of diaphragmatic ultrasound and chest radiography to determine endotracheal tube position in a pediatric emergency department. Pediatrics 123: e1039-1044.

17. Uya A, Spear D, Patel K Okada P, Sheeran P, et al. (2012) Can novice sonographers accurately locate an endotracheal tube with a saline-filled cuff in a cadaver model? A pilot study. Acad Emerg Med 19: 361-364.

18. Tessaro MO, Salant EP, Arroyo AC, Haines LE, Dickman E, et al. (2015) Tracheal rapid ultrasound saline test (T.R.U.S.T.) for confirming correct endotracheal tube depth in children. Resuscitation 89: 8-12.

19. Rabb MF, Szmuk P (2007) The difficult pediatric airway. In: Benumof's Airway Management: Principles and Practice. 2nd edition. Elsevier's Health Sciences.

20. Koshy T, Misra S, Chatterjee N, Dharan B (2016) Accuracy of a chest X-ray based method for predicting the depth of insertion of endotracheal tubes in pediatric patients undergoing cardiac surgery. Journal of Cardiothoracic and Vascular Anesthesia 30: 947-953.

21. Thayyil S, Nagakumar P, Gowers H, Sinha A (2008) Optimal Endotracheal Tube Tip Position in Extremely Premature Infants. Am J Perinatol 25: 13-17.

22. F Onyekwulu, T Prasad, R Nagarajan (2010) Appropriate Placement O Endotracheal Tubes In Pediatric Cardiac Patients. The Internet Journal of Anesthesiology.

23. Jaeel P, Sheth M, Nguyen J (2017) Ultrasonography for endotracheal tube position in infants and children. European Journal of Pediatrics 176: 293 300.

24. Magee P, Tooley M (2011) Airway management devices. In: The Physics, Clinical Measurement and Equipment of Anaesthetic Practice for the FRCA. 2nd edition. Oxford University Press, Oxford.

25. Combes X, Schauvliege F, Peyrouset O, Motamed C, Kirov K, et al. (2001) Intracuff pressure and tracheal morbidity: influence of filling with saline during nitrous oxide anesthesia. Anesthesiology 95: 1120-1124 